\title{
Global Encoding for Long Chinese Text Summarization
}

\author{
XUEFENG XI and ZHOU PI, Suzhou University of Science and Technology \\ GUODONG ZHOU, Soochow University
}

\begin{abstract}
Text summarization is one of the significant tasks of natural language processing, which automatically converts text into a summary. Some summarization systems, for short/long English, and short Chinese text, benefit from advances in the neural encoder-decoder model because of the availability of large datasets. However, the long Chinese text summarization research has been limited to datasets of a couple of hundred instances. This article aims to explore the long Chinese text summarization task. To begin with, we construct a first large-scale, long Chinese text summarization corpus, the Long Chinese Summarization of Police Inquiry Record Text (LCSPIRT). Based on this corpus, we propose a sequence-to-sequence (Seq2Seq) model that incorporates a global encoding process with an attention mechanism. Our model achieves a competitive result on the LCSPIRT corpus compared with several benchmark methods.
\end{abstract}

CCS Concepts: • Computing methodologies $\rightarrow$ Discourse, dialogue and pragmatics; Information extraction; Language resources;

Additional Key Words and Phrases: Text summarization, long Chinese text, corpus building

\section{ACM Reference format:}

Xuefeng Xi, Zhou Pi, and Guodong Zhou. 2020. Global Encoding for Long Chinese Text Summarization. ACM Trans. Asian Low-Resour. Lang. Inf. Process. 19, 6, Article 84 (October 2020), 17 pages.

https://doi.org/10.1145/3407911

\section{INTRODUCTION}

Text summarization is one of the significant tasks of natural language processing, which automatically converts text into a summary. It can save search time and simplify the search process. In the current information explosion era, text summarization is particularly essential for improving the efficiency of knowledge discovery tasks.

Text summarization has two typical methods: extractive summarization and abstractive summarization. The former is summarized directly from the source text and usually selects a complete sentence at a time. The latter can generate different words and phrases, rather than copy them from the source text. Based on a sequence-to-sequence (Seq2Seq) model that corresponds to neural machine translation [Bahdanau et al. 2014], many abstractive summarization methods have performed well on short English text datasets [Cheng and Lapata 2016; Chopra et al. 2016;

This research has been supported by the National Natural Science Foundation of China under grants 61673290, 61876217, 61672371, 61876121; the Science \& Technology Development Project of Suzhou under grant SYG201817; and the Innovative Team of Jiangsu Province under grant XYDXX-086.

Authors' addresses: X. Xi and Z. Pi, Suzhou University of Science and Technology; emails: xfxi@usts.edu.cn, pizhou@ post.usts.edu.cn; G. Zhou (corresponding author), Soochow University, 1 ShiZi Street, Suzhou, Jiangsu, China; email: gdzhou@suda.edu.cn.

Permission to make digital or hard copies of part or all of this work for personal or classroom use is granted without fee provided that copies are not made or distributed for profit or commercial advantage and that copies bear this notice and the full citation on the first page. Copyrights for third-party components of this work must be honored. For all other uses, contact the owner/author(s).

(c) 2020 Copyright held by the owner/author(s).

2375-4699/2020/10-ART84

https://doi.org/10.1145/3407911 
Luong et al. 2015; Rush et al. 2015; See et al. 2017], long English text datasets [Celikyilmaz et al. 2018; Cohan et al. 2018; Dong et al. 2018; Fabbri et al. 2019; Gehrmann et al. 2018; Kingma and Ba 2019; Li et al. 2018; Liu et al. 2018; Liu and Lapata 2019; Zhou et al. 2018], and short Chinese text datasets [Hu et al. 2015; Li et al. 2020; Lin et al. 2018; Wei et al. 2019]. However, it is infrequent to explore text summarization on long Chinese text due to the lack of high-quality long Chinese text summarization datasets.

This article aims to explore the long Chinese text summarization task. The three major contributions of this article are as follows: (a) The long Chinese text summarization dataset in the police domain is initially constructed. It plays an essential role in the information extraction task for public social security. (b) A Seq2Seq summarization model for long Chinese text is proposed. To the best of our knowledge, this work is the first attempt to address the neural text summarization of single, long Chinese documents. (c) For different lengths of Chinese text, we analyze different processing models and provide a suitable model selection strategy.

The rest of this article is organized as follows: Section 2 discusses related works. Section 3 describes our LCSPIRT corpus. Section 4 presents a Seq2Seq model for long Chinese text summarization. In Section 5, we express our experiments and evaluate our approach using the datasets from LCSPIRT corpus. Section 6 analyzes and discusses the results. Section 7 concludes our work.

\section{RELATED WORK}

As an important branch of natural language processing, text summarization has developed for decades. In recent years, with the development of computer hardware, the application of machine learning in text summarization had been further developed. Rush first applied the deep learning method to text summarization [Rush et al. 2015]. They used a local attention-based model to generate summarization words according to input sentences and used Rouge to score them. However, their method was limited in the size of input documents and summaries. Based on their work, Nallapati also added the attention mechanism in the encoder-decoder model [Nallapati et al. 2016]. When decoding, the generated words of each decoding layer were taken from the vocabulary created by the original document. At the same time, to introduce new words, they added a layer in the input to use word2vec to obtain the synonym structure of the context. Their approaches depended on the generator/pointer model with both input words and context-based synonyms as input [Luong et al. 2015]. For long text summarization, Ling adopted two layers of hierarchical attention mechanism [Ling and Rush 2017], one of which used attention mechanism to select one or more critical words from input documents and then fed them to the second layer through a sequence pair sequential model. They used reinforcement learning to train the attention model. This method realized the processing of long text sequences, but its accuracy was not good. Cohan proposed an abstract summarization model [Cohan et al. 2018], which included a hierarchical encoder for simulating discourse structure and a perceptual decoder for generating abstracts. Based on Bidirectional Encoder Representations from Transformers (BERT [Devlin et al. 2019]), Liu propose a general framework for both extractive and abstractive models [Liu and Lapata 2019].

Compared with the English text summarization work, the research on short Chinese text summarization has also developed in recent years. LCSTS was the first large Chinese short text dataset [Hu et al. 2015]. Gu proposed a new model COPYNET for summarizing short articles [Gu et al. 2016]. After integrating a new copying mechanism into the conventional approach generating word in the decoder, this model can put the subsequences selected from the input sequence at the appropriate position of the output sequence. Due to text summarization relying on multiple reading of source text and multi-level general information, Ma [Ma and Sun 2017] found that there are apparent hierarchical phenomena in text. But there were no corresponding multiple structures in the end-to-end model. To address this issue, they proposed a multi-level encoder 
to obtain different levels of text information. Li [Li et al. 2017] proposed a new text summarization framework based on the end-to-end encoder-decoder model, which added a deep recursive generation encoder (DRGN). Hou proposed an LSTM-based model to identify high-quality text summary pairs for building large-scale paired datasets [Hou et al. 2017]. Ma proposed a hierarchical end-to-end model [Ma et al. 2018], which classified sentences as a further generalization of text summarization. Lin [Lin et al. 2018] proposed a framework based on global encoding, which used convolution gating unit to control the information flow from the encoder to decoder according to the global information of input context. Wei [Wei et al. 2019] proposed a regularization approach to the sequence-to-sequence model for the Chinese social media summarization task, which could improve the semantic consistency. Based on a double attention pointer network, $\mathrm{Li}[\mathrm{Li}$ et al. 2020] proposed an encoder-decoder model achieved higher summarization performance on the CNN/Daily Mail dataset and the LCSTS dataset.

\section{LCSPIRT CORPUS}

Because there are few long Chinese text summarization datasets, we use police inquiry record text (PIRT) to construct a large-scale, long Chinese text summarization corpus called Long Chinese Summarization of PIRT (LCSPIRT). This corpus includes two sub-corpora: LCSPIRT in a Dialogue Manner (LCSPIRT-DM) and LCSPIRT in a Paragraph Manner (LCSPIRT-PM). LCSPIRT-DM consists of 38,500 text-summary pairs that we, together with the local police, manually tagged in which the dialogue manner was adopted. Subsequently, we construct LCSPIRT-PM by removing the question part from LCSPIRT-DM. Each sub-corpus contains 38,500 text-summary pairs; hence, the two sub-corpora contain a total of 77,000 text-summary pairs. The text in the corpus is desensitized to ensure privacy.

Compared to LCSPIRT-DM, the text in LCSPIRT-PM has a more compact distribution of keywords than LCSPIRT-DM, because there is no QuestionX (e.g., Q2 removed from Table 1) separation between Answer 1 (e.g., A1 in Table 1) and Answer2 (e.g., A2 in Table 1). Two examples are shown in Table 1 . This corpus ${ }^{1}$ has been released, and we expect that this work will promote advances in long Chinese text summarization.

\subsection{Statistics and Analysis}

LCSPIRT-DM and LCSPIRT-PM both mainly pair the source text and summary. Their length distributions are shown in Figure 1. The lengths of the source text in the LCSPIRT-DM corpus are mainly concentrated in the intervals of 500-1,400 characters. Although the lengths of the source text in LCSPIRT-PM corpus are less than LCSPIRT-DM due to removing the question part, it still mainly concentrates on between 200 and 1,200 characters. That indicates that our corpus belongs to a type of long text corpora, which is different from other short text summarization corpora.

For LCSPIRT-DM and LCSPIRT-PM, there is the same summary of the source text, because the existence of questions has nothing to do with the semantics of the summary. The length of the summary ranges from 49 to 115 characters, and it is centered at 70 characters.

\subsection{Other Corpus}

As mentioned, there are few corpora of Chinese long text summarization. In the case of the Chinese text summarization dataset, a short Chinese text summarization corpus called LCSTS was constructed by $\mathrm{Hu}$ [Hu et al. 2015]. The data in LCSTS corpus was from the Chinese microblogging website Sina Weibo. It includes pairs of short text-summary in Chinese. Among which Part II(10,666 pairs) and Part III(1,106 pairs) are manually labeled except for Part I(2,400,591 pairs).

\footnotetext{
${ }^{1}$ LCSPIRT corpus is currently available at http://eie.usts.edu.cn/prj/NLPoSUST/LcsPIRT.htm.
} 
Table 1. Two Examples of Text-summary Pair in LCSPIRT-DM and LCSPIRT-PM

\begin{tabular}{|c|c|c|}
\hline Item & A source text in Chinese & A translation in English \\
\hline $\begin{array}{l}\text { Text in a } \\
\text { Dialogue } \\
\text { Manner }\end{array}$ & $\begin{array}{l}\text { 问 1: 我们是**市公安局**派出所的 } \\
\text { 民警 } \\
\text { (出示工作证件), } \\
\text { 现依法对你进行询问, } \\
\text { 请你如实回答问题, } \\
\text { 对与本案无关的问题, } \\
\text { 你有拒绝回答的权利, } \\
\text { 你听清楚了吗? } \\
\text { 答 } 1: \text { 听清楚了。 } \\
\text { 问 2: 你今天拨打 } 110 \text { 所为何事? } \\
\text { 答 2: 电瓶被盗。 } \\
\text { 问 3: 案发经过? } \\
\text { 答 } 3: 2017 \text { 年11月 } 26 \text { 日 } 17 \text { 时 } \\
\text { 至 } 22 \text { 时, } \\
\text { 停放在国贸大厦南侧的停车场 } \\
\text { 电动车被盗。 } \\
\text { 问 4: 损失情况? } \\
\text { 答4: 电瓶被盗, } 48 \mathrm{v}, 2014 \text { 年购买 } \\
\text { 花了 } 500 \text { 元。 } \\
\text { 问5: 你还有什么要补充的吗? } \\
\text { 答5: 没有。 } \\
\text { 问 6: 你以上说的是否属实? } \\
\text { 答6: 属实。 }\end{array}$ & $\begin{array}{l}\text { Q1: We are the policemen of the XX police } \\
\text { station of XX Public Security Bureau } \\
\text { (showing their certificates). } \\
\text { Now we will ask you some questions } \\
\text { in the name of the law. Please answer the ones } \\
\text { truthfully. You have the right to refuse to } \\
\text { answer questions unrelated to this case. } \\
\text { Do you understand? } \\
\text { A1: Yes, I do. } \\
\text { Q2: Why do you call } 110 \text { today? } \\
\text { A2: My battery had been stolen. } \\
\text { Q3: Could you please describe how it happened? } \\
\text { A3: From } 17: 00 \text { to } 22: 00 \text { on November } 26,2017, \\
\text { Some things on the electric bicycle were stolen. } \\
\text { It was parking on the south side of } \\
\text { the international trade building. } \\
\text { Q4: What was lost? } \\
\text { A4: The battery was stolen. Its capacity is } 48 \mathrm{~V} \text {. } \\
\text { It cost } 500 \text { CNY in } 2014 \text {. } \\
\text { Q5: Anything else? } \\
\text { A5: No, thank you. } \\
\text { Q6: Is that true? } \\
\text { A6: Yes, it is. }\end{array}$ \\
\hline $\begin{array}{l}\text { Text in } \\
\text { a Para- } \\
\text { graph } \\
\text { Manner }\end{array}$ & $\begin{array}{l}\text { 答1: 听清楚了。 } \\
\text { 答 2: 电瓶被盗。 } \\
\text { 答 } 3: 2017 \text { 年11月 } 26 \text { 日 } 17 \text { 时 } \\
\text { 至 } 22 \text { 时, } \\
\text { 停放在国贸大厦南侧的停车场 } \\
\text { 电动车被盗。 } \\
\text { 答4: 电瓶被盗, } 48 \mathrm{v}, \\
\text { 2014年购买花了 } 500 \text { 元。 } \\
\text { 答5: 没有。 } \\
\text { 答6: 属实。 }\end{array}$ & $\begin{array}{l}\text { A1: Yes, I do. } \\
\text { A2: My battery had been stolen. } \\
\text { A3: From 17:00 to 22:00 on November 26, 2017, } \\
\text { Some things on the electric bicycle were stolen. } \\
\text { It was parking on the south side of } \\
\text { the international trade building. } \\
\text { A4: The battery was stolen. Its capacity is } 48 \mathrm{~V} \text {. } \\
\text { It cost } 500 \text { CNY in } 2014 \text {. } \\
\text { A5: No, thank you. } \\
\text { A6: Yes, it is. }\end{array}$ \\
\hline Summary & $\begin{array}{l}2017 \text { 年11月 } 26 \text { 日 } 17 \text { 时 } \\
\text { 到 } 2017 \text { 年11月 } 26 \text { 日 } 22 \text { 时 } \\
\text { 晚上马女士 } \\
\text { 在国贸大厦南侧 } \\
\text { 被嫌疑人以其它盗窃手段 } \\
\text { 窃走电瓶车电瓶1个。 }\end{array}$ & $\begin{array}{l}\text { From } 17: 00, \text { November } 26,2017 \\
\text { to } 22: 00, \text { November } 26,2017, \\
\text { one battery of Ms. Ma's electric bicycle } \\
\text { was stolen by the suspect using } \\
\text { other method on the south side of } \\
\text { the international trade building. }\end{array}$ \\
\hline
\end{tabular}

The statistics of the LCSTS and our LCSPIRT are shown in Table 2. Additionally, Figure 2 shows the length distribution of the text and summary from LCSPIRT and LCSTS. For both text and summary, the length in LCSPIRT is much higher than LCSTS.

\section{OUR MODEL}

\subsection{Model Architecture}

For the long Chinese text summarization task, we propose an improved Seq2Seq model framework inspired by Lin [Lin et al. 2018]. Our model framework takes a long Chinese text as input and outputs a summary. As shown in Figure 3, it primarily consists of the following four components: data preprocessing, encoder, global encoding unit (GEU), and decoder. Based on the outputs from the gated recurrent unit (GRU) encoder [Cho et al. 2014], GEU refines the representation of 

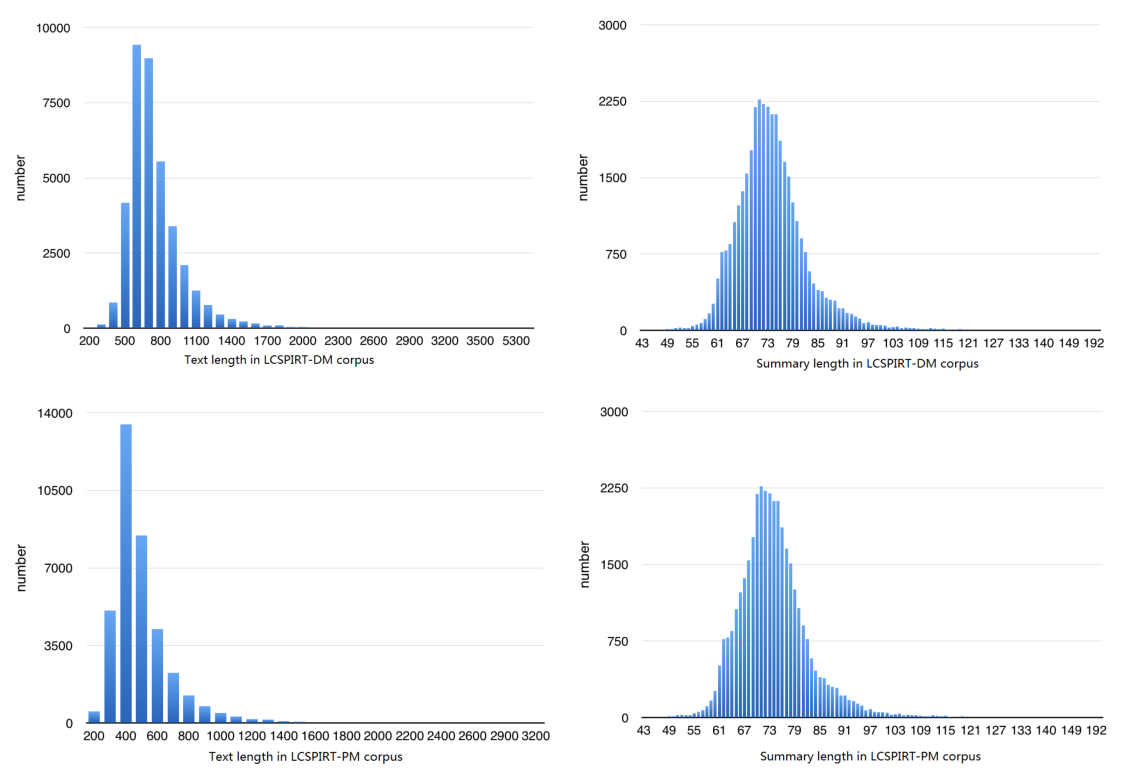

Fig. 1. Length statistics diagram of LCSPIRT corpus.

Table 2. Statistics of LCSPIRT and Other Corpora

\begin{tabular}{ccccc}
\hline \multirow{2}{*}{ Corpus } & Pairs labelled & \multicolumn{2}{c}{ Text } & Summary \\
& manually & Avg. sentences & Avg. characters & Avg. characters \\
\hline LCSTS & 11,772 & 10.13 & 108.8 & 19 \\
LCSPIRT-DM & 38,500 & 35.28 & 684.3 & 75 \\
LCSPIRT-PM & 38,500 & 22.43 & 451.3 & 75 \\
\hline
\end{tabular}

the source context using a convolutional neural network (CNN), attention mechanism, and gated unit.

Compared with Lin's model [Lin et al. 2018], our proposed improved model has two main changes. One is that Chinese words are used as an embedding vector representation instead of Chinese characters. The second change is that a GRU module is used instead of a long short-term memory (LSTM) module as the encoder-decoder unit. The details of the techniques are introduced in the following subsections, and their advantages are discussed in Section 6.2.

\subsection{Data Preprocessing}

The function of the data preprocessing component is to convert the input source text into a vector representation. After receiving the original text, we first use the Jieba tool ${ }^{2}$ to perform Chinese word segmentation. The obtained words then form a dictionary. Simultaneously, we input all the original text to train the word embedding unit. Finally, the original text is transformed into a word vector through the word dictionary and word embedding unit.

\footnotetext{
${ }^{2}$ It is currently available at https://www.github.com/fxsjy/jieba.
} 

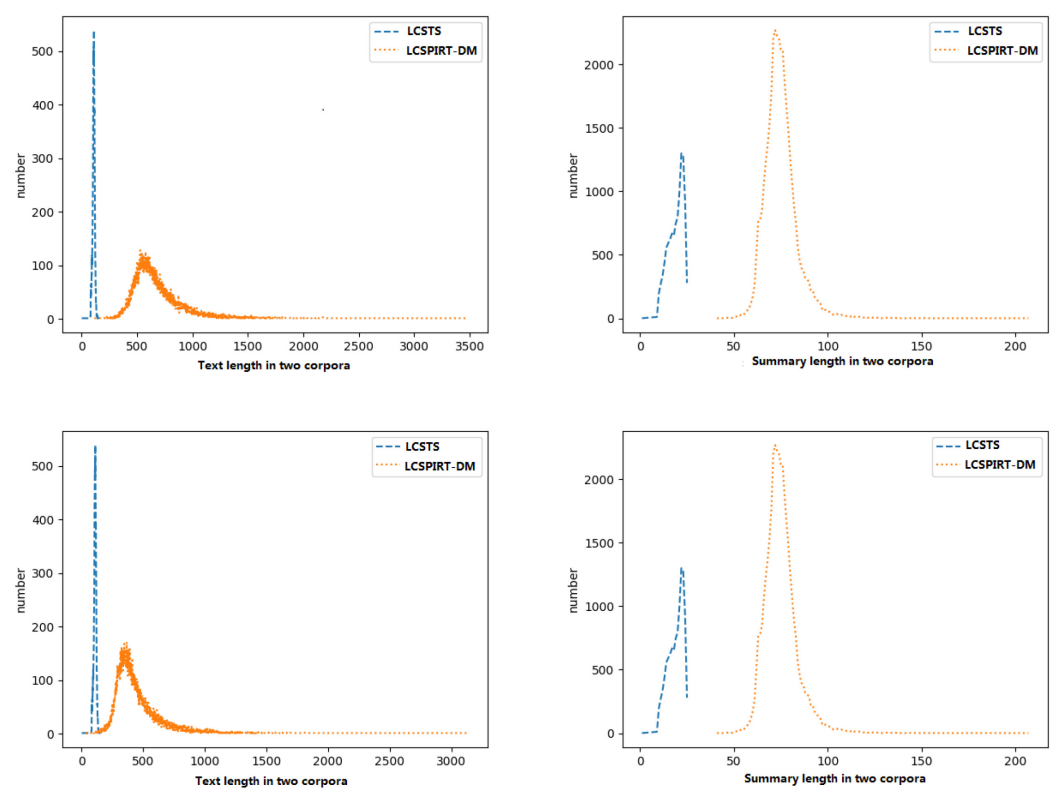

Fig. 2. Length comparison diagram of LCSPIRT and other corpora.

\subsection{Encoder}

We use the GRU as an encoder. This encoder receives the word embedding of each word from the source text sequentially and generates hidden states at each time-step. All hidden states build a matrix $H$, which is input to the next GEU. The last hidden state $h_{t}$ contains information about the entire source text. This becomes the initial hidden state of the subsequent processing component, the decoder (see Section 4.5):

$$
\begin{gathered}
z_{t}=\sigma\left(W_{z} \cdot\left[h_{t-1}, x_{t}\right]\right), \\
r_{t}=\sigma\left(W_{r} \cdot\left[h_{t-1}, x_{t}\right]\right), \\
\tilde{h}_{t}=\tanh \left(W \cdot\left[r_{t} * h_{t-1}, x_{t}\right]\right), \\
h_{t}=\left(1-z_{t}\right) * h_{t-1}+z_{t} * \tilde{h}_{t},
\end{gathered}
$$

where $x_{t}$ is an input vector, $h_{t}$ is an output vector, $z_{t}$ is an update gate vector, and $r_{t}$ is a reset gate vector. $W$ is a parameter matrix.

\subsection{Global Encoding Unit}

The GEU integrates global information and an attention mechanism to implement a re-encoding function. This component includes three modules: feature extraction via a CNN, global attention, and a gated unit.

Feature Extraction via a CNN. We use one-dimensional convolution to extract the $n$-gram features. The convolution block is described in detail as follows:

$$
\tilde{g}_{m}=\operatorname{ReLU}\left(W \cdot\left[h_{m-k / 2}, \ldots, h_{m+k / 2}\right]+b\right),
$$

where $R e L U$ refers to the non-linear activation function rectified linear unit. Based on this convolution block, we implement a structure similar to inception [Szegedy et al. 2016].

Global Attention. In addition to the new representations generated by the CNN module, we further implement an attention mechanism on these representations to dig out the global correlations. 


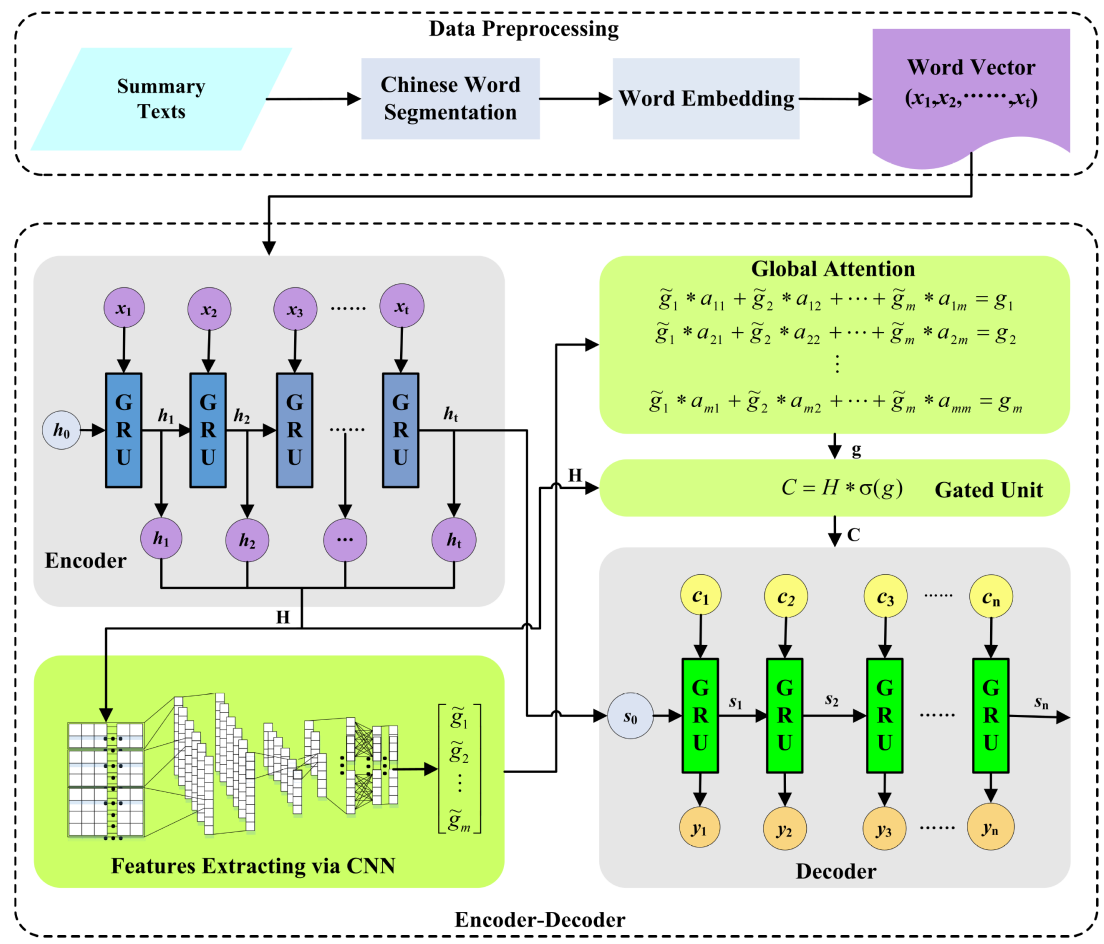

Fig. 3. Overview of our model. The GRU in the encoder is shown in blue and the GRU in the decoder is shown in green. At each encoding timestep, the encoder forms a context vector $h_{t}$, which encodes the relevant source context word vector $\left(x_{t}\right)$. Matrix $H$ is used as an input to the $\mathrm{CNN}$, which extracts $n$-gram features. Then global attention on these $n$-gram features identifies the global correlations and outputs $g$. Hidden states $H$ and $g$ are used as two inputs to the gated unit (a logistic sigmoid function) to perform global encoding. Finally, the decoder (using the green GRU) generates summary $y$ word-by-word according to the global encoding result (matrix $C$ ) and the relevant encoding information $\left(s_{0}\right.$ is initialized as the last hidden state $h_{t}$ from the encoder).

The attention mechanism in Transformer Model [Vaswani et al. 2017] is a function that includes a query (Q), a set of Key (K), and value (V) pairs [Lin et al. 2018]:

$$
\operatorname{Attention}(Q, K, V)=\operatorname{softmax}\left(\frac{Q K^{\mathrm{T}}}{\sqrt{d_{k}}}\right) V \text {. }
$$

Gated Unit. A further step is to set a gate based on the generation from the CNN and attention mechanism module $g$ for the source representations $H$ from the GRU encoder, and the $*$ represents element-wise multiplication. where:

$$
C=H * \sigma(g) .
$$

The logistic sigmoid function $\sigma$ outputs a vector between 0 and 1 in each dimension. If the value is close to 0 , the gate deletes most of the information on the corresponding dimension of the source representation. If the value is close to 1 , the gate retains most of the information. Thus, the gated unit can perform global encoding on the encoder outputs. 


\subsection{Decoder}

As shown in Figure 3, the decoder is used to decode the intermediate semantic vector $C$. According to $C$ and the final hidden state $h_{t}$, the green GRU (decoder) generates summary sequence words $\left(y_{1}, y_{2}, \ldots, y_{n}\right)$. Similar to the encoder, the GRU is also used as a decoder. The details of the decoding process are described below:

$$
\begin{gathered}
z_{n}=\sigma\left(W_{z} \cdot\left[s_{n-1}, c_{n}\right]\right), \\
r_{n}=\sigma\left(W_{r} \cdot\left[s_{n-1}, c_{n}\right]\right), \\
\tilde{s}_{n}=\tanh \left(W \cdot\left[r_{n} * s_{n-1}, c_{n}\right]\right), \\
s_{n}=\left(1-z_{n}\right) * s_{n-1}+z_{n} * \tilde{s}_{n}, \\
y_{n}=\operatorname{softmax}\left(W_{d} \cdot s_{n}\right) .
\end{gathered}
$$

\subsection{Training}

Given the parameters $\theta$ and source text $x$, the model generates a summary $y$. The learning process minimizes the negative log-likelihood between the generated summary $y$ and reference summary $\tilde{y}:$

$$
\mathfrak{L}=-\frac{1}{T} \sum_{t=1}^{T} \sum_{n=1}^{N} p\left(\tilde{y}_{n}^{(t)} \mid y_{<n}^{(t)}, x^{(t)}, \theta\right),
$$

where $T$ and $N$ are the length of the generated summary and reference summary, respectively. The $t$ and $n$ are variables. Here the loss function is equivalent to maximizing the conditional probability of summary $y$ given parameters $\theta$ and source text sequence $x$.

\section{EXPERIMENT}

In the following, we introduce 10 datasets and our experimental settings, in addition to the baseline models that we used for comparison.

\subsection{Datasets}

As mentioned earlier in this article, LCSPIRT-DM is a large-scale, long Chinese text summarization corpus that we constructed that consists of 38,500 text-summary pairs. The original texts were longer than 70 Chinese characters, and the summaries were created manually. Compared with LCSPIRT-DM, the original text in the LCSPIRT-PM corpus does not contain the question part; otherwise, there is no difference between LCSPIRT-DM and LCSPIRT-PM. The datasets for these two corpora are denoted by $\mathrm{DM}_{\text {Origin }}$ and $\mathrm{PM}_{\text {Origin }}$, respectively. The length statistics of the source texts for the two subsets are shown in Figure 1.

To verify the performance of different models for different Chinese characters in a long text, we first split the texts in $\mathrm{PM}_{\text {Origin }}$, which was sorted by length, into four equal subsets, where each subset was around $9 \mathrm{~K}$ summary pairs, denoted by $\mathrm{PM}_{A}, \mathrm{PM}_{B}, \mathrm{PM}_{C}$, and $\mathrm{PM}_{D}$, respectively. As shown in Table 3, the maximum lengths of the texts in the four $\mathrm{PM}_{x}$ datasets are with 328, 396, 508, and 3,136 characters, respectively. In this study, the distance represents the gap between the maximum and minimum length of the text.

To verify whether the question part affects on summarization performance, we restored the question in the four $\mathrm{PM}_{x}$ datasets to construct the $\mathrm{DM}_{A}, \mathrm{DM}_{B}, \mathrm{DM}_{C}$, and $\mathrm{DM}_{D}$ datasets. Table 4 illustrates the character lengths of the four $\mathrm{DM}_{x}$ datasets. 
Table 3. Statistics of Our LCSPIRT-PM Datasets

\begin{tabular}{cccccc}
\hline Length & \multicolumn{5}{c}{ Datasets } \\
(characters) & $\mathrm{PM}_{\text {Origin }}$ & $\mathrm{PM}_{A}$ & $\mathrm{PM}_{B}$ & $\mathrm{PM}_{C}$ & $\mathrm{PM}_{D}$ \\
\hline Max. of text & 3,136 & 328 & 396 & 508 & 3,136 \\
Min. of text & 69 & 69 & 328 & 396 & 508 \\
Distance of text & 3,067 & 259 & 68 & 112 & 2,628 \\
Avg. of text & 451.3 & 281.6 & 362.5 & 446.4 & 715.0 \\
Avg. of summary & 75.0 & 73.4 & 74.7 & 75.7 & 76.2 \\
\hline
\end{tabular}

Table 4. Statistics of Our LCSPIRT-DM Datasets

\begin{tabular}{cccccc}
\hline $\begin{array}{c}\text { Length } \\
\text { (characters) }\end{array}$ & $\mathrm{DM}_{\text {Origin }}$ & $\mathrm{DM}_{A}$ & $\mathrm{DM}_{B}$ & $\mathrm{DM}_{C}$ & $\mathrm{DM}_{D}$ \\
\hline Max. of text & 3,487 & 1,052 & 1,130 & 2,606 & 3,487 \\
Min. of text & 114 & 114 & 438 & 510 & 639 \\
Distance of text & 3,373 & 938 & 692 & 2,096 & 2,848 \\
Avg. of text & 684.3 & 476.2 & 581.0 & 688.9 & 991.2 \\
Avg. of summary & 75.0 & 73.4 & 74.7 & 75.7 & 76.2 \\
\hline
\end{tabular}
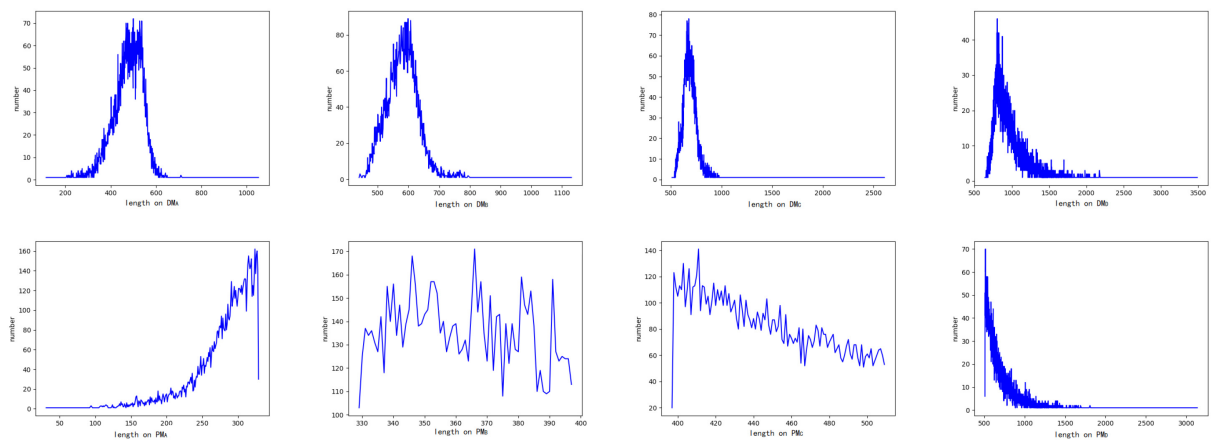

Fig. 4. Length statistics of the texts from datasets.

The length statistics of the source texts for the eight subsets of $\mathrm{PM}_{x}$ and $\mathrm{DM}_{x}$ are shown in Figure 4. Finally, we followed previous research by splitting all the datasets into training $(80 \%)$, validation(10\%), and testing(10\%) datasets.

\subsection{Baseline Methods}

To evaluate the performance of the model on our datasets, we implemented six baseline models: TextRank, ${ }^{3}$ LexRank, ${ }^{4}$ Pointer-Generator, ${ }^{5}$ HiMap, ${ }^{6}$ Seq2Seq/GEU+LSTM/C, ${ }^{7}$ and Seq2Seq/LSTM/C.

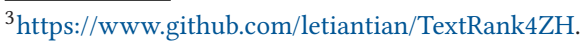

${ }^{4}$ https://www.github.com/crabcamp/lexrank.

${ }^{5}$ http://www.github.com/abisee/pointer-generator.

${ }^{6}$ https://www.github.com/Alex-Fabbri/Multi-News.

${ }^{7}$ https://www.github.com/lancopku/Global-Encoding.
} 
TextRank is a graph-based ranking model for extractive summarization. It does not require deep linguistic knowledge, nor domain or language-specific annotated corpora [Mihalcea and Tarau 2004]. TextRank is often used as a benchmark model for processing small, short text datasets because of its simplicity.

LexRank is a stochastic graph-based ranking model for calculating the relative importance of sentences in a text unit [Erkan and Radev 2004]. The most important sentences in a text are identified for text summarization.

Pointer-Generator is a hybrid generation model for text summarization. It can not only generate words from word-list but also copy words from raw material [See et al. 2017]. That solves the problem that the traditional Seq2Seq model cannot create out-of-vocabulary (OOV). Besides, it uses coverage to track the words that have been summarized to solve the problem of repeated generation.

HiMap combines the traditional summarization model with a standard single document summarization model [Fabbri et al. 2019]. It was proposed for the first large-scale multi-document summarization news dataset. The model incorporates a maximal marginal relevance algorithm into the pointer-generator network and achieves outstanding results in the multi-document summarization task.

Seq2Seq/GEU+LSTM/C is a Seq2Seq model with GEU and LSTM module based on Chinese characters $(\mathrm{C})$, which outperformed state-of-the-art models for short Chinese text summarization [Lin et al. 2018].

Seq2Seq/LSTM/C is a traditional Seq2Seq model with LSTM module based on Chinese characters (C), which is implemented by removing the GEU component from the Seq2Seq/GEU+LSTM/C model.

\subsection{Benchmark Methods}

Using our improved model framework, we constructed four Seq2Seq models based on Chinese words (W) to evaluate performance on our datasets: Seq2Seq/LSTM/W, Seq2Seq/GRU/W, Seq2Seq/GEU+LSTM/W, and Seq2Seq/GEU+GRU/W.

These four models are all Seq2Seq models. Compared with the baseline model Seq2Seq/GEU+LSTM/C, there are two differences between our four models. The first is that the four models use Chinese words as the word embedding rather than Chinese characters. The second difference is the type of neural network unit that is used as the encoder or decoder. The Seq2Seq/LSTM/W model uses LSTM as a fundamental component of the encoder and decoder. By comparison, the Seq2Seq/GRU/W model uses the GRU instead of LSTM. Our proposed Seq2Seq/GEU+GRU/W model adopts the GRU as the encoder and decoder with a GEU. Similar to this Seq2Seq/GEU+GRU/W model, the Seq2Seq/GEU+LSTM/W model replaces GRU with LSTM as the encoder and decoder.

\subsection{Setting}

We implemented our experiments using PyTorch ${ }^{8}$ on an NVIDIA 2080Ti GPU. The word embedding dimension and number of hidden units were both 128. In all experiments, the batch size was set to 64. We used the Adam optimizer [Kingma and Ba 2014] with the default setting $\alpha=0.001$, $\beta_{1}=0.9, \beta_{2}=0.999$, and $\epsilon=1 \times 10^{-8}$. The learning rate was halved at each epoch. Gradient clipping was applied with the range $[-10,10]$. As shown in Figure 5 , the loss function values in all models tended to converge when the epoch approached 20, so we set the epoch to 20 .

\footnotetext{
$\overline{{ }^{8} \text { https://pytorch.org/. }}$
} 

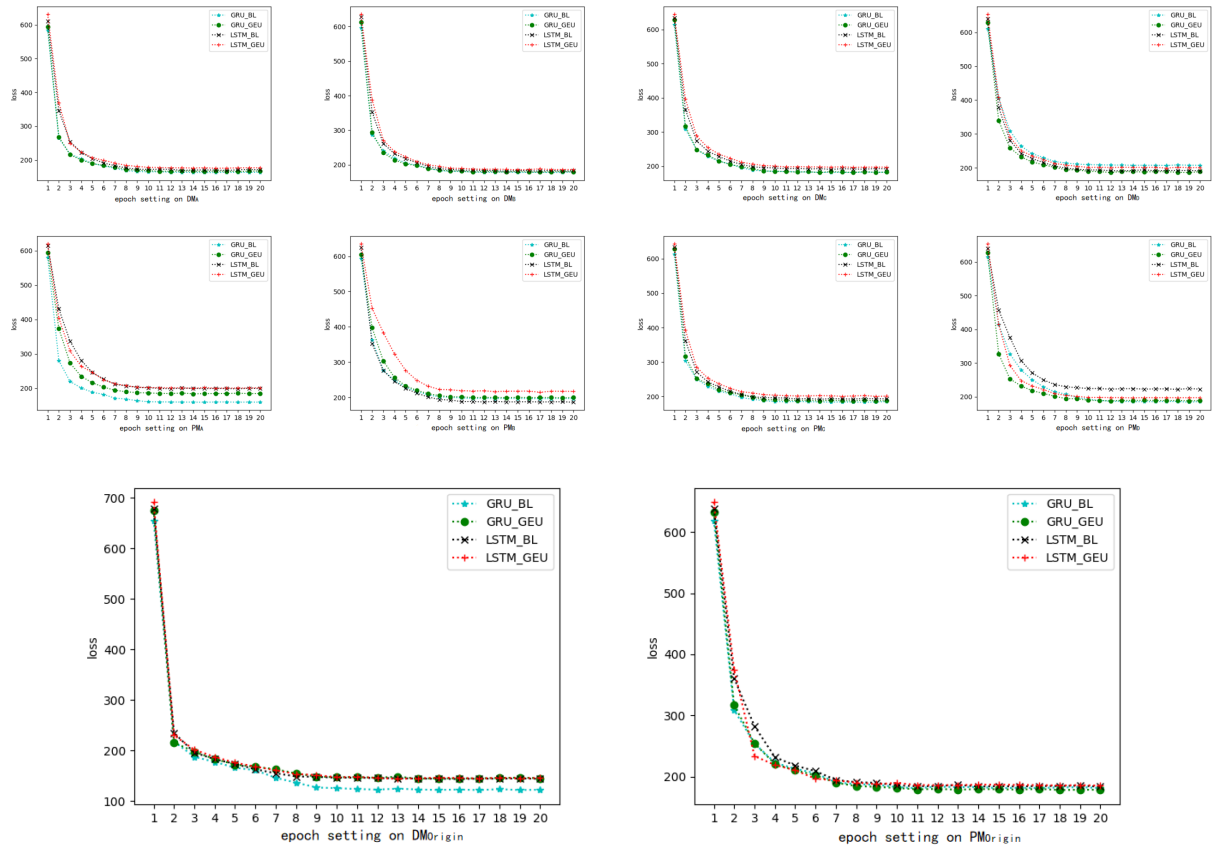

Fig. 5. Losses of all models with epoch steps.

Some evaluation methods (ROUGE [Lin and Hovy 2003], BLEU [Papineni et al. 2002], MoverScore [Zhao et al. 2019]) are adopted in text summarization. As we all know, the evaluation of large-scale summarization models is costly and cumbersome. Considering the cost and popularity, we chose the ROUGE score to evaluate the performance of our models. The ROUGE score is used to calculate the degree of overlap between a generated summary and reference, including the number of $n$-grams. The F1 scores of ROUGE-1, ROUGE-2, and ROUGE-L were used as the evaluation metrics.

\section{ANALYSIS}

\subsection{Results}

The results of 65 experiments on 10 datasets using different models are shown in Tables 5-9. Among the results, Table 5 shows the first group of experiments (17 experiments on two complete corpus datasets, $\mathrm{DM}_{\text {Origin }}$ and $\mathrm{PM}_{\text {Origin }}$ ). Tables 6-9 show the second group of experiments (48 experiments on eight subsets of two corpora, $\mathrm{DM}_{A \sim D}$ and $\mathrm{PM}_{A \sim D}$ ).

In the first group of experiments, the Seq2Seq models outperform other models. For the $\mathrm{PM}_{\text {Origin }}$ dataset, the Seq2Seq/GEU+GRU/W model obtained the highest scores, 53.93 (Rouge-

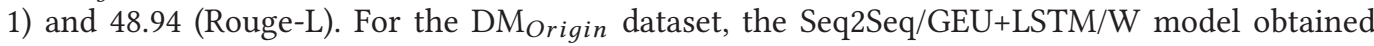
the highest scores, 43.35 (Rouge-1) and 36.97 (Rouge-L). By contrast, the other models received lower scores. Since their performances are much lower than the Seq2Seq models, we did not use them in subsequent experiments. Additionally, we have observed a few inconsistent numbers in Table 5. There are two examples: One is the Seq2Seq/LSTM/C model: It has high Rouge- 2 but very low Rouge-L. The other case is Seq2Seq/GEU+GRU/W model: It has excellent results on $\mathrm{PM}_{\text {Origin }}$ dataset but bad ROUGE scores on $\mathrm{DM}_{\text {Origin }}$ dataset. It seems hard to explain thoroughly, because the models are not interpretable. But we can explain it in some ways. Because the Seq2Seq/LSTM/C 
Table 5. Comparison of Results on DMorigin and PMorigin Datasets

\begin{tabular}{lcccccc}
\hline \multirow{2}{*}{ Models } & \multicolumn{3}{c}{ Dataset DMorigin } & \multicolumn{3}{c}{ Dataset PM Origin } \\
& Rouge-1 & Rouge-2 & Rouge-L & Rouge-1 & Rouge-2 & Rouge-L \\
\hline TextRank & $*$ & $*$ & $*$ & 11.24 & 1.5 & 10.84 \\
LexRank & 4.44 & 0.94 & 3.88 & $*$ & $*$ & $*$ \\
HiMap/C & 21.6 & 10.68 & 17.76 & $*$ & $*$ & $*$ \\
Point-Generator/C & 24.89 & 12.88 & 16.78 & $*$ & $*$ & $*$ \\
Point-Generator/W & 40.03 & 25.14 & 31.7 & $*$ & $*$ & $*$ \\
Seq2Seq/LSTM/C & 34.72 & 32.52 & 21.56 & 35.07 & 32.76 & 21.91 \\
Seq2Seq/GEU+LSTM/C & 33.06 & 23.60 & 19.50 & 15.29 & 9.41 & 6.65 \\
Seq2Seq/LSTM/W & 26.41 & 10.97 & 15.7 & 48.09 & 17.89 & 43.31 \\
Seq2Seq/GRU/W & 39.06 & 29.77 & 28.98 & 49.79 & 22.79 & 47.36 \\
Seq2Seq/GEU+LSTM/W & $\mathbf{4 3 . 3 5}$ & 13.32 & $\mathbf{3 6 . 9 7}$ & 48.92 & 14.99 & 42.36 \\
Seq2Seq/GEU+GRU/W & 26.13 & 11.44 & 15.88 & $\mathbf{5 3 . 9 3}$ & 22.56 & $\mathbf{4 8 . 9 4}$ \\
\hline
\end{tabular}

Table 6. Comparison of Results on $\mathrm{DM}_{A}$ and $\mathrm{PM}_{A}$ Datasets

\begin{tabular}{lcccccc}
\hline \multirow{2}{*}{ Models } & \multicolumn{3}{c}{ Dataset DM $_{\boldsymbol{A}}$} & \multicolumn{3}{c}{ Dataset PM $_{\boldsymbol{A}}$} \\
& Rouge-1 & Rouge-2 & Rouge-L & Rouge-1 & Rouge-2 & Rouge-L \\
\hline Seq2Seq/LSTM/C & 34.76 & 23.92 & 20.18 & 33.59 & 23.63 & 19.52 \\
Seq2Seq/GEU+LSTM/C & 35.52 & 23.65 & 20.55 & 34.55 & 24.02 & 20.13 \\
Seq2Seq/LSTM/W & $\mathbf{5 9 . 2 8}$ & $\mathbf{3 1 . 6 0}$ & $\mathbf{5 6 . 3 5}$ & 57.06 & 27.73 & 54.03 \\
Seq2Seq/GRU/W & 56.50 & 28.88 & 53.69 & $\mathbf{5 7 . 1 6}$ & $\mathbf{2 8 . 7 6}$ & $\mathbf{5 4 . 6 9}$ \\
Seq2Seq/GEU+LSTM/W & 58.67 & 30.62 & 56.20 & 51.82 & 23.96 & 48.36 \\
Seq2Seq/GEU+GRU/W & 56.39 & 28.13 & 53.64 & 52.37 & 25.33 & 49.14 \\
\hline
\end{tabular}

Table 7. Comparison of Results on $\mathrm{DM}_{B}$ and $\mathrm{PM}_{B}$ Datasets

\begin{tabular}{lcccccc}
\hline \multirow{2}{*}{ Models } & \multicolumn{3}{c}{ Dataset DM $_{\boldsymbol{B}}$} & \multicolumn{3}{c}{ Dataset PM $_{\boldsymbol{B}}$} \\
& Rouge-1 & Rouge-2 & Rouge-L & Rouge-1 & Rouge-2 & Rouge-L \\
\hline Seq2Seq/LSTM/C & 34.31 & 23.39 & 19.92 & 33.93 & 23.13 & 19.13 \\
Seq2Seq/GEU+LSTM/C & 32.7 & 23.03 & 19.19 & 44.84 & 16.5 & 33.36 \\
Seq2Seq/LSTM/W & $\mathbf{5 6 . 1 9}$ & $\mathbf{2 8 . 4 0}$ & 53.40 & 54.01 & 25.23 & 51.41 \\
Seq2Seq/GRU/W & 52.68 & 25.60 & 49.91 & $\mathbf{5 6 . 8 0}$ & $\mathbf{2 9 . 7 8}$ & $\mathbf{5 3 . 9 8}$ \\
Seq2Seq/GEU+LSTM/W & 56.12 & 28.19 & $\mathbf{5 3 . 4 7}$ & 52.47 & 24.13 & 49.86 \\
Seq2Seq/GEU+GRU/W & 53.71 & 25.41 & 51.20 & 51.58 & 23.06 & 48.89 \\
\hline
\end{tabular}

Table 8. Comparison of Results on $\mathrm{DM}_{C}$ and $\mathrm{PM}_{C}$ Datasets

\begin{tabular}{lcccccc}
\hline \multirow{2}{*}{ Models } & \multicolumn{3}{c}{ Dataset DM } & \multicolumn{3}{c}{ Dataset PM } \\
& Rouge-1 & Rouge-2 & Rouge-L & Rouge-1 & Rouge-2 & Rouge-L \\
\hline Seq2Seq/LSTM/C & 32.98 & 22.89 & 19.10 & 32.84 & 24.49 & 19.17 \\
Seq2Seq/GEU+LSTM/C & 32.46 & 22.89 & 18.88 & 33.64 & 23.04 & 19.09 \\
Seq2Seq/LSTM/W & 51.86 & 24.89 & 49.60 & 51.37 & 23.18 & 48.44 \\
Seq2Seq/GRU/W & $\mathbf{5 3 . 2 0}$ & $\mathbf{2 5 . 7 2}$ & $\mathbf{5 0 . 3 5}$ & $\mathbf{5 2 . 6 5}$ & $\mathbf{2 5 . 7 3}$ & $\mathbf{5 0 . 0 5}$ \\
Seq2Seq/GEU+LSTM/W & 50.42 & 22.69 & 48.67 & 49.62 & 23.07 & 47.23 \\
Seq2Seq/GEU+GRU/W & 51.56 & 23.50 & 49.32 & 49.53 & 22.68 & 46.88 \\
\hline
\end{tabular}


Table 9. Comparison of Results on $\mathrm{DM}_{D}$ and $\mathrm{PM}_{D}$ Datasets

\begin{tabular}{lcccccc}
\hline \multirow{2}{*}{ Models } & \multicolumn{3}{c}{ Dataset DM $_{D}$} & \multicolumn{3}{c}{${\text { Dataset } \text { PM }_{D}}$} \\
& Rouge-1 & Rouge-2 & Rouge-L & Rouge-1 & Rouge-2 & Rouge-L \\
\hline Seq2Seq/LSTM/C & 34.43 & 22.69 & 19.98 & 33.54 & 23.59 & 19.33 \\
Seq2Seq/GEU+LSTM/C & 27.46 & 15.11 & 14.88 & 44.36 & 20.38 & 32.75 \\
Seq2Seq/LSTM/W & 51.03 & $\mathbf{2 3 . 0 8}$ & $\mathbf{4 8 . 2 2}$ & 53.38 & 22.64 & 48.61 \\
Seq2Seq/GRU/W & 49.76 & 20.50 & 45.41 & $\mathbf{5 4 . 3 4}$ & $\mathbf{2 6 . 9 3}$ & $\mathbf{5 0 . 9 3}$ \\
Seq2Seq/GEU+LSTM/W & $\mathbf{5 2 . 0 7}$ & 20.87 & 47.07 & 38.49 & 8.84 & 32.19 \\
Seq2Seq/GEU+GRU/W & 47.69 & 16.81 & 42.18 & 44.01 & 14.30 & 37.90 \\
\hline
\end{tabular}

model used Chinese characters as an embedding vector representation, it is more conducive to capture the semantics of a single character. Then it is easier to generate a suitable short-characters summary. That is good for getting a large Rouge-2, but not for Rouge-L. For the other example, a significant factor may be the different manner of the two datasets. Dialogue manner in $\mathrm{DM}_{\text {Origin }}$ dataset leads to a broader separation of the semantic relationship than paragraph manner in $\mathrm{PM}_{\text {Origin }}$ dataset. The wider the semantic relationship, the more difficult it is to be captured by the Seq2Seq/GEU+GRU/W model.

The second group of experiments' aim is to evaluate the effect of different lengths of source text on performance for the Seq2Seq models. First, the models based on Chinese words performance better than the ones found on Chinese characters. As shown in Tables 6-9, around 50 points of Rouge- 1 obtained by the former is much higher than approximately 30 points of Rouge- 1 earned by the latter. Second, the stability performance of Seq2Seq models based on Chinese words is always obtained on different length datasets. However, the performance of the Seq2Seq/GEU+LSTM/W model on the $\mathrm{PM}_{D}$ dataset is much lower than its performance on the other length datasets.

Generally, on the complete corpus datasets $\left(\mathrm{DM}_{\text {Origin }}\right.$ and $\left.\mathrm{PM}_{\text {Origin }}\right)$, our models with GEU significantly outperformed state-of-the-art models. On different subsets of the corpus, the performance of the two traditional Seq2Seq word-based models was consistently better than that of the two models with GEU. However, the latter two word-based models were very close in performance to the former two word-based models. Thus, all seq2Seq word-based models had excellent performance.

\subsection{Discussion}

First, compared with the baseline system, the most significant difference in our proposed model is that Chinese words are used as an embedding vector representation rather than Chinese characters. As shown in Figure 6, the Chinese word-based embedding vector representation model was better than the character-based embedding vector representation model for our text summarization task. Figure 6 is a comparison of the results on the Chinese character-based model (Seq2Seq/GEU+LSTM/C) and word-based model (Seq2Seq/GEU+LSTM/W was also one of our best models). The three sub-graphs at the top of Figure 6 show the comparison of the results on five datasets from the LCSPIRT-DM corpus using three evaluation indicators: Rouge-1, Rouge-2, and Rouge-L, respectively. Additionally, the three sub-graphs at the bottom of Figure 6 show the comparison of the results on five datasets from the other corpus, LCSPIRT-PM. Almost all the brown columns that represent the results of the word-based model exceed the blue columns that represent the results of the character-based model. The reason may be that the police inquiry record text dataset we considered contained a large number of standard addresses, standard times, and other conventional representations. For these representations, existing Chinese word segmentation technology has better semantic acquisition performance. Thus, it is easier to obtain accurate 

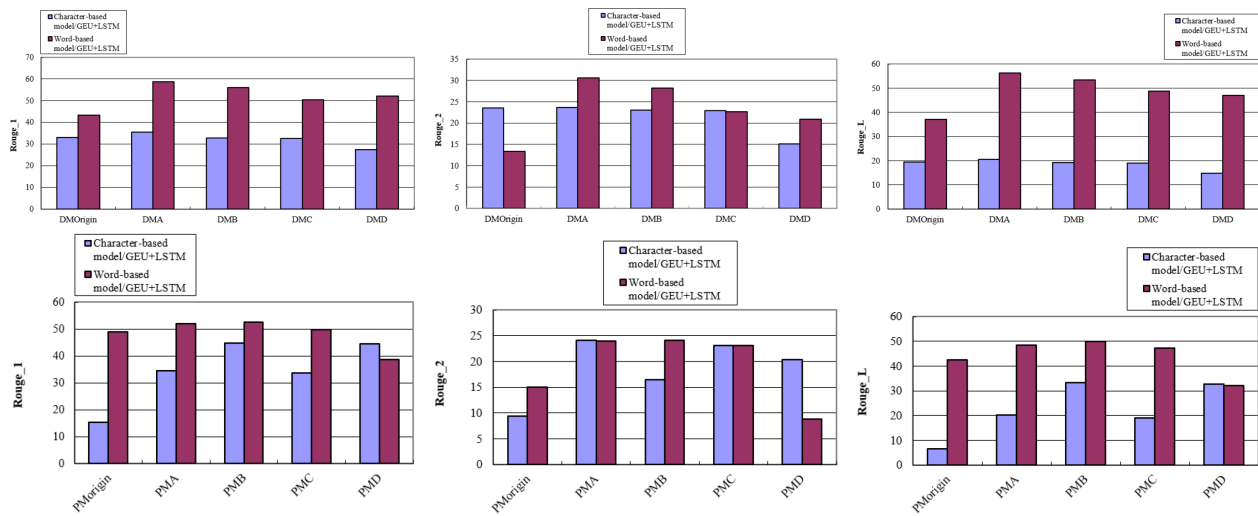

Fig. 6. Comparison of the results on Chinese character-based model and word-based model according to different evaluation criterias in LCSPIRT-DM corpus and LCSPIRT-PM corpus. Two typical models (Seq2Seq/GEU+LSTM/C and Seq2Seq/GEU+LSTM/W) are used as objects of comparison, which are the same except for the difference in the form of embedding representation.

semantic abstract representations through the vector representation space constructed, followed word segmentation. Furthermore, these word-based models achieved better abstract extraction performance.

Second, the overall results of the experiment show that the Seq2Seq word-based model with GEU had better performance. According to the different characteristics of the datasets, we also analyzed the performance of different models, observed some interesting phenomena, and summarized the following related strategies.

Relationship between Model and Variance of the Input Length. The variance of the input length represents the gap between the maximum and minimum length of the input text. By synthetically analyzing all the experimental results, we observed the following two interesting phenomena: (a) The model with GEU was good at processing datasets with a big variance of the length. As shown in Table 5, we found that the Seq2Seq/GEU+GRU and Seq2Seq/GEU+GRU models were optimized on the $\mathrm{DM}_{\text {Origin }}$ and $\mathrm{PM}_{\text {Origin }}$ datasets, respectively. Additionally, these two datasets are only the same type of dataset with a big variance. (b) By contrast, traditional Seq2Seq models seem to be better at processing datasets with a small variance of the length. As seen from Tables 6-9, traditional Seq2Seq models without GEU tended to perform better on datasets with a small variance.

By synthesizing the above analysis, we can devise a selection strategy as follows: If the dataset that we need to process has a relatively big variance of the length, then we adopt the Seq2Seq model with GEU. Otherwise, we prefer traditional Seq2Seq models.

Relationship between the Model and Dataset in a Dialogue/Paragraph Manner. In our datasets, the source text for the data in the LCSPIRT-DM corpus was a type of data in a dialogue manner. By contrast, what came from the LCSPIRT-PM corpus was not in a dialogue manner but a kind of paragraph, which was transformed from the LCSPIRT-DM corpus by removing the question part. From the results, we observed another interesting phenomenon: For the dataset in a dialogue manner, the LSTM model had advantages over the GRU model; for the dataset in a paragraph manner, the GRU model was better than the LSTM model. For instance, as shown in Table 5, the Seq2Seq/GEU+LSTM/W model was in first place with 43.35 (Rouge-1) and 36.97 (Rouge-L) on the DM $\mathrm{Drigin}_{\text {in }}$ dataset, whereas the Seq2Seq/GEU+GRU/W model achieved the best results on the 
Table 10. Selection Strategy of the Seq2Seq Models

\begin{tabular}{lcccc}
\hline \multirow{2}{*}{ Models } & \multicolumn{2}{c}{ Big-Variance } & \multicolumn{2}{c}{ Small-Variance } \\
& Dialogue & Paragraph & Dialogue & Paragraph \\
\hline Seq2Seq/LSTM/W & & & \\
Seq2Seq/GRU/W & & & \\
Seq2Seq/GEU+LSTM /W & & & \\
Seq2Seq/GEU+GRU/W & & & \\
\hline
\end{tabular}

$\mathrm{PM}_{\text {Origin }}$ dataset. Similar cases occurred in the three subsequent results tables: Table 6, Table 7 , and Table 9.

Relationship between Performance and Dataset in a Dialogue/Paragraph Manner. For the same model, we consider the different effects of different datasets on performance. By synthetically analyzing all the experimental results, we observed the following two interesting phenomena: (a) With the same model and same dataset with a significant variance of the input length, the performance of the dataset in a paragraph manner was better than that of a dataset in a dialogue manner. As shown in Table 5, we found that the performance of the four models on the PM $\mathrm{P}_{\text {Origin }}$ dataset was better than that on the $\mathrm{DM}_{\text {Origin }}$ dataset. One possible reason is that, for datasets with a significant variance of the input length, the dataset in a paragraph manner shortened the semantic distance and achieved better semantic capture performance. (b) With the same model and same dataset with a small variance of the input length, there was no apparent difference in the different manner of corpora.

Based on the analysis of the above characteristics, we present the strategies shown in Table 10. For example, when we need to process a small variance of the input length and dialogue style source text, we first identify the corresponding column unit location from Table 10, and then retrieve the column unit content to find the star tag. At this point, the model corresponding to the row corresponding to the star tag is the model that we can recommend for application on this input datasets. Just like human reading, when encountering different types and lengths of documents, people assume the scene to be processed in advance and adopt different processing strategies. For instance, people tend to pay more attention to the event development route of the text for fiction, whereas people tend to pay more attention to details and facts for scientific research papers.

\section{CONCLUSION}

To our best knowledge, our proposed work is the first attempt at addressing the text summarization of single, long Chinese documents via the neural encoder-decoder model. We used the police inquiry record text to construct a large-scale, long Chinese text summarization corpus, LCSPIRT. For this corpus, we presented some neural Seq2Seq models with GEU. This unit effectively summarizes long and unstructured Chinese documents. Our experimental results are encouraging, and our new corpus can help the NLP community to explore long Chinese text summarization further.

Additionally, we explored a variety of neural network models on some datasets with varying lengths and different structures of text. Through the comparative analysis of the results, we proposed a model selection strategy for datasets to be processed according to different characteristics. We believe that this selection strategy is helpful for text summarization.

\section{REFERENCES}

Dzmitry Bahdanau, KyungHyun Cho, and Yoshua Bengio. 2014. Neural machine translation by jointly learning to align and translate. arXiv:arXiv preprint arXiv:1409.0473 (2014).

Asli Celikyilmaz, Antoine Bosselut, Xiaodong He, and Yejin Choi. 2018. Deep communicating agents for abstractive summarization. In Proceedings of the Conference of the North American Chapter of the Association for Computational Linguistics: Human Language Technologies, Volume 1 (Long Papers). 1662-1675. 
Jianpeng Cheng and Mirella Lapata. 2016. Neural summarization by extracting sentences and words. In Proceedings of the 54th Meeting of the Association for Computational Linguistics. 484-494.

Kyunghyun Cho, Bart van Merrienboer, Dzmitry Bahdanau, and Yoshua Bengio. 2014. On the properties of neural machine translation: Encoder-decoder approaches. In Proceedings of the 8th Workshop on Syntax, Semantics and Structure in Statistical Translation (SSST'14). 103-111.

Sumit Chopra, Michael Auli, and Alexander M. Rush. 2016. Abstractive sentence summarization with attentive recurrent neural networks. In Proceedings of the Conference of the North American Chapter of the Association for Computational Linguistics: Human Language Technologies. 93-98.

Arman Cohan, Franck Dernoncourt, Doo Soon Kim, Trung Bui, Seokhwan Kim, Walter Chang, and Nazli Goharian. 2018. A discourse-aware attention model for abstractive summarization of long documents. In Proceedings of the Conference of the North American Chapter of the Association for Computational Linguistics: Human Language Technologies, Volume 2 (Short Papers). 615-621.

Jacob Devlin, Ming-Wei Chang, Kenton Lee, and Kristina Toutanova. 2019. BERT: Pre-training of deep bidirectional transformers for language understanding. In Proceedings of the Conference of the North American Chapter of the Association for Computational Linguistics: Human Language Technologies, Volume 1 (Long and Short Papers). 4171-4186.

Yue Dong, Yikang Shen, Eric Crawford, Herke van Hoof, and Jackie Chi Kit Cheung. 2018. BanditSum: Extractive summarization as a contextual bandit. In Proceedings of the Conference on Empirical Methods in Natural Language Processing. 3739-3748.

Günes Erkan and Dragomir R. Radev. 2004. Lexrank: Graph-based lexical centrality as salience in text summarization. 7 . Arti. Intell. Res. 22 (2004), 457-479.

Alexander R. Fabbri, Irene Li, Tianwei She, Suyi Li, and Dragomir R. Radev. 2019. Multi-news: A large-scale multi-document summarization dataset and abstractive hierarchical model. In Proceedings of the 57th Meeting of the Association for Computational Linguistics. 1074-1084.

Sebastian Gehrmann, Yuntian Deng, and Alexander Rush. 2018. Bottom-up abstractive summarization. In Proceedings of the Conference on Empirical Methods in Natural Language Processing. 4098-4109.

Jiatao Gu, Zhengdong Lu, Hang Li, and Victor O. K. Li. 2016. Incorporating copying mechanism in sequence-to-sequence learning. In Proceedings of the 54th Meeting of the Association for Computational Linguistics. 1631-1640.

Yongshuai Hou, Yang Xiang, Buzhou Tang, Qingcai Chen, Xiaolong Wang, and Fangze Zhu. 2017. Identifying high quality document-summary pairs through text matching. Information 8, 2 (2017), 64.

Baotian Hu, Qingcai Chen, and Fangze Zhu. 2015. LCSTS: A large scale Chinese short text summarization dataset. In Proceedings of the Conference on Empirical Methods in Natural Language Processing. 1967-1972.

Diederik P. Kingma and Jimmy Ba. 2014. Adam: A method for stochastic optimization. arXiv:arXiv preprint arXiv:1412.6980 (2014).

Diederik P. Kingma and Jimmy Ba. 2019. Scoring sentence singletons and pairs for abstractive summarization. arXiv: arXiv preprint arXiv:1906.00077 (2019).

Piji Li, Wai Lam, Lidong Bing, and Zihao Wang. 2017. Deep recurrent generative decoder for abstractive text summarization. In Proceedings of the Conference on Empirical Methods in Natural Language Processing. 2091-2100.

Wei Li, Xinyan Xiao, Yajuan Lyu, and Yuanzhuo Wang. 2018. Improving neural abstractive document summarization with explicit information selection modeling. In Proceedings of the Conference on Empirical Methods in Natural Language Processing. 1787-1796.

Zhixin Li, Zhi Peng, Suqin Tang, Canlong Zhang, and Huifang Ma. 2020. Text summarization method based on double attention pointer network. IEEE Access 8 (2020), 11279-11288.

Chin-Yew Lin and Eduard Hovy. 2003. Automatic evaluation of summaries using n-gram co-occurrence statistics. In Proceedings of the Human Language Technology Conference of the North American Chapter of the Association for Computational Linguistics.

Junyang Lin, Xu Sun, Shuming Ma, and Qi Su. 2018. Global encoding for abstractive summarization. In Proceedings of the 56th Meeting of the Association for Computational Linguistics (Volume 2). 163-169.

Jeffrey Ling and Alexander Rush. 2017. Coarse-to-fine attention models for document summarization. In Proceedings of the Workshop on New Frontiers in Summarization. 33-42.

Peter J. Liu, Mohammad Saleh, Etienne Pot, Ben Goodrich, Ryan Sepassi, Lukasz Kaiser, and Noam Shazeer. 2018. Generating Wikipedia by summarizing long sequences. arXiv:arXiv preprint arXiv:1801.10198 (2018).

Yang Liu and Mirella Lapata. 2019. Text summarization with pretrained encoders. In Proceedings of the Conference on Empirical Methods in Natural Language Processing and the 9th International foint Conference on Natural Language Processing (EMNLP-IFCNLP). 3721-3731.

Thang Luong, Ilya Sutskever, Quoc Le, Oriol Vinyals, and Wojciech Zaremba. 2015. Addressing the rare word problem in neural machine translation. In Proceedings of the 53rd Meeting of the Association for Computational Linguistics and the 7th International foint Conference on Natural Language Processing (Volume 1: Long Papers), Vol. 1. 11-19. 
Shuming Ma and Xu Sun. 2017. A semantic relevance based neural network for text summarization and text simplification. arXiv:arXiv preprint arXiv:1710.02318 (2017).

Shuming Ma, Xu Sun, Junyang Lin, and Xuancheng Ren. 2018. A hierarchical end-to-end model for jointly improving text summarization and sentiment classification. In Proceedings of the 27th International foint Conference on Artificial Intelligence. AAAI Press, 4251-4257.

Rada Mihalcea and Paul Tarau. 2004. Textrank: Bringing order into text. In Proceedings of the Conference on Empirical Methods in Natural Language Processing.

Ramesh Nallapati, Bowen Zhou, Cicero dos Santos, Caglar Gulcehre, and Bing Xiang. 2016. Abstractive text summarization using sequence-to-sequence RNNs and beyond. In Proceedings of the 20th SIGNLL Conference on Computational Natural Language Learning. 280-290.

Kishore Papineni, Salim Roukos, Todd Ward, and Weijing Zhu. 2002. BLEU: A method for automatic evaluation of machine translation. In Proceedings of the 40th Meeting of the Association for Computational Linguistics. 311-318.

Alexander M. Rush, Sumit Chopra, and Jason Weston. 2015. A neural attention model for abstractive sentence summarization. In Proceedings of the Conference on Empirical Methods in Natural Language Processing. 379-389.

Abigail See, Peter J. Liu, and Christopher D. Manning. 2017. Get to the point: Summarization with pointer-generator networks. In Proceedings of the 55th Meeting of the Association for Computational Linguistics. 1073-1083.

Christian Szegedy, Vincent Vanhoucke, Sergey Ioffe, Jon Shlens, and Zbigniew Wojna. 2016. Rethinking the inception architecture for computer vision. In Proceedings of the IEEE Conference on Computer Vision and Pattern Recognition. 2818-2826.

Ashish Vaswani, Noam Shazeer, Niki Parmar, Jakob Uszkoreit, Llion Jones, Aidan N. Gomez, Łukasz Kaiser, and Illia Polosukhin. 2017. Attention is all you need. In Proceedings of the Conference on Advances in Neural Information Processing Systems. 5998-6008.

Bingzhen Wei, Xuancheng Ren, Yi Zhang, Xiaoyan Cai, Qi Su, and Xu Sun. 2019. Regularizing output distribution of abstractive Chinese social media text summarization for improved semantic consistency. ACM Trans. Low-resour. Lang. Inf. Proc. 18, 3 (2019), 1-15.

Wei Zhao, Maxime Peyrard, Fei Liu, Yang Gao, Christian M. Meyer, and Steffen Eger. 2019. MoverScore: Text generation evaluating with contextualized embeddings and earth mover distance. In Proceedings of the Conference on Empirical Methods in Natural Language Processing and the 9th International foint Conference on Natural Language Processing. 563578.

Qingyu Zhou, Nan Yang, Furu Wei, Shaohan Huang, Ming Zhou, and Tiejun Zhao. 2018. Neural document summarization by jointly learning to score and select sentences. In Proceedings of the 56th Meeting of the Association for Computational Linguistics. 654-663.

Received August 2019; revised April 2020; accepted June 2020 